\title{
Stable Teleoperation with Communication Unreliabilities and Approximate Human/Environment Dynamics Knowledge
}

\author{
Iason Vittorias and Sandra Hirche
}

\begin{abstract}
This paper introduces a novel control approach for teleoperation systems to achieve finite gain $\mathscr{L}_{2}$-stability under communication unreliabilities. It is assumed that the communication block satisfies a small gain condition, as for example constant time delay and properly handled packet loss do. The proposed approach, a generalization of the scattering transformation, takes advantage of dissipative properties of human arm, environment, and/or manipulator dynamics. It is shown that transparency can be substantially improved compared to the conventional scattering transformation approach. Simulations and experimental results validate the proposed approach.
\end{abstract}

\section{INTRODUCTION}

A teleoperation system allows the human to manipulate in remote, inaccessible, dangerous, or scaled environments. Audio, vision, and haptic data, are exchanged between a human system interface and a teleoperator, see Fig. 1. From a control point of view, the haptic control loop, where motion and force data are exchanged between the master and the slave manipulator, is very challenging as it is closed over a communication network, e.g. the Internet. The communication network introduces unreliabilities such as (time-varying) time delay and packet loss, which do not only distort the human haptic perception of the remote environment but potentially destabilizing the overall system. This paper focuses on finite gain $\mathscr{L}_{2}$-stability of a haptic feedback system under such communication unreliabilities.

Over the past 20 years control approaches based on the passivity framework and the scattering transformation have been developed in order to stabilize a teleoperation system in the presence of communication unreliabilities. Inspired by power transmission lines, the scattering transformation is proposed in [1] where arbitrary large constant time-delay is addressed. The equivalent wave variables notation first appears in [17]. The scattering transformation approach has been extended to stabilize with packet loss, see e.g. [2], [7] and with time-varying time delay [20]. The major reason for the success of the passivity formalism in teleoperation is that it can cope with the largely unknown, nonlinear and time-varying human arm and environment dynamics, which typically can assumed to be passive [11]. However, the passivity framework is known to be conservative resulting in a distorted display of the remote environment properties [9]. Relaxing the passivity conservatism by using knowledge on the human dynamics is performed in [3] using a natural

I. Vittorias and S. Hirche are with the Institute of Automatic Control Engineering (LSR), Technische Universität München, Arcisstrasse 21, 80290 München, Germany. vittorias@mytum. de, hirche@tum.de

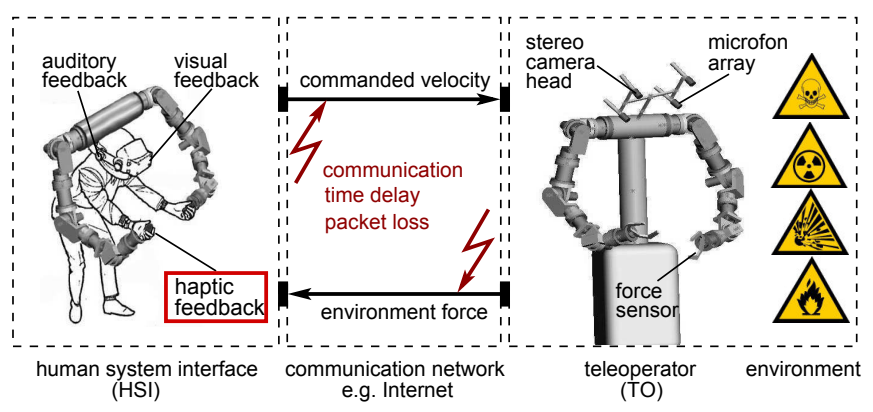

Fig. 1. Multimodal teleoperation system

admittance control (NAC) scheme. Stability analysis is performed with a classical robust control framework, however, without considering a teleoperation setup and communication unreliabilities. Gillespie in [6] models the human as a second-order linear time-invariant system and proposes an observer scheme. However, stability can be guaranteed only for constant user impedances, which is known to be not the case [18]. Moreover, the exploitation of the mechanical properties of the environment can also greatly improve the performance of the robotic system, see [5]. In summary, the largely unknown and time-varying nature of human operator and environment dynamics has continued to pose a challenge for the design of guaranteed stable haptic systems.

The contribution of this paper - in contrast to existing approaches - is the use of approximate knowledge of the damping properties of the human arm, the controlled manipulators, and/or the environment for stabilizing control design of a teleoperation system with communication unreliabilities. In fact, we can show that these subsystems are QSR-dissipative [19], which will be exploited to the benefit of transparency. The approach is based on the generalized scattering transformation (GST) [10], [15], [16]. It applies to QSR-dissipative systems, ensuring finite gain $\mathscr{L}_{2}$-stability for arbitrary small gain network operators, e.g. constant time delay or appropriately handled packet loss. A transparency analysis of the proposed scheme for the constant delay case shows improved performance, in terms of displayed mechanical properties, compared to the standard scattering transformation.

The remainder of the paper is organized as follows: in section II the background for the dissipativity-based modeling of our system, in section III, is presented. The generalized scattering transformation is studied in section IV followed by the corresponding transparency analysis in section $\mathrm{V}$. Experimental results are presented in section VI. 


\section{DISSIPATIVITY THEORY BACKGROUND}

Consider a system described by $h: \dot{x}=f(x, u), y=h(x, u)$ where $x \in \mathfrak{R}^{n}, u \in \mathfrak{R}^{p}, y \in \mathfrak{R}^{q}$ are the state, input and output vectors respectively, and $f(0,0)=h(0,0)=0$.

Definition 1: A dynamical system $\Sigma: \dot{x}=f(x, u)$, $y=h(x, u)$ is called QSR-dissipative if there exist a positive semi-definite function $V: \mathfrak{R}^{n} \rightarrow \mathfrak{R}_{+}$such that for each admissible $u$ and each $t \geq 0$

$$
V(x(t))-V(x(0)) \leq \int_{0}^{t}\left[\begin{array}{ll}
u & y
\end{array}\right]^{T} P\left[\begin{array}{l}
u \\
y
\end{array}\right] \mathrm{d} \tau,
$$

with dissipativity matrix

$$
P=\left[\begin{array}{cc}
Q & S \\
S^{T} & R
\end{array}\right]
$$

where $Q \in \mathfrak{R}^{p \times p}, Q \in \mathfrak{R}^{q \times q}$ and $S \in \mathfrak{R}^{p \times q}$. For the ease of notation the dependencies of $u, y$ from time $\tau$ are not explicitly written.

Input-feedforward-output-feedback passive systems (IF-OFP), a subclass of QSR-dissipative systems with $u, y \in \mathfrak{R}^{p}$, are represented by the choice

$$
P=\left[\begin{array}{cc}
-\delta I & \frac{1}{2} I \\
\frac{1}{2} I & -\varepsilon I
\end{array}\right],
$$

$\delta, \varepsilon \in \Re$, and $I$ the unity matrix. The system is called lossless if $\delta=\varepsilon=0$, output-feedback strictly passive $(\operatorname{OFP}(\varepsilon))$ if $\delta=0$ and $\varepsilon>0$, and input-feedforward strictly passive $(\operatorname{IFP}(\delta))$ if $\delta>0$ and $\varepsilon=0$. If one or both of the values $\delta, \varepsilon$ are negative, then there is a shortage of passivity.

Feedback interconnected IFP and OFP systems still exhibit IF-OFP properties as shown below.

Lemma 1: The negative feedback interconnection of $\operatorname{OFP}\left(\varepsilon_{1}\right)$ system $\Sigma_{1}$ with $\operatorname{IFP}\left(\delta_{2}\right)$ system $\Sigma_{2}$ is $\operatorname{OFP}\left(\varepsilon_{1}+\delta_{2}\right)$. Proof: The system $\Sigma_{1}$ is $\operatorname{OFP}\left(\varepsilon_{1}\right)$ thus

$$
V_{1}(x(t))-V_{1}(x(0)) \leq \int_{0}^{t} u_{1}^{T} y_{1}-\varepsilon_{1} y_{1}^{T} y_{1} \mathrm{~d} \tau,
$$

whereas for the $\operatorname{IFP}\left(\delta_{2}\right)$ system $\Sigma_{2}$

$$
V_{2}(x(t))-V_{2}(x(0)) \leq \int_{0}^{t} u_{2}^{T} y_{2}-\delta_{2} u_{2}^{T} u_{2} \mathrm{~d} \tau,
$$

holds, where $u_{1}, u_{2}$ and $y_{1}, y_{2}$ are the respective inputs and outputs, see Fig. 2(a). For the negative feedback interconnection of those two systems considering the compound storage function $V=V_{1}+V_{2}$ gives

$$
\begin{aligned}
& V(x(t))-V(x(0)) \leq \int_{0}^{t} u_{1}^{T} y_{1}-\varepsilon_{1} y_{1}^{T} y_{1}+u_{2}^{T} y_{2}-\delta_{2} u_{2}^{T} u_{2} \mathrm{~d} \tau \\
& =\int_{0}^{t}\left(e_{1}-y_{2}\right)^{T} y_{1}-\varepsilon_{1} y_{1}^{T} y_{1}+y_{1}^{T} y_{2}-\delta_{2} y_{1}^{T} y_{1} \mathrm{~d} \tau \\
& =\int_{0}^{t} e_{1}^{T} y_{1}-\left(\varepsilon_{1}+\delta_{2}\right) y_{1}^{T} y_{1} \mathrm{~d} \tau .
\end{aligned}
$$

where $u_{1}=e_{1}-y_{2}, e_{1}$ being the input to the overall system, and $u_{2}=y_{1}$ hold. The output of the overall system is $y_{1}$.

Lemma 2: Consider the $\operatorname{IFP}\left(\delta_{1}\right)$ system $\Sigma_{1}$ and the $\operatorname{OFP}\left(\varepsilon_{2}\right)$ system $\Sigma_{2}$ with $\delta_{1} \geq 0$ and $\delta_{1}+\varepsilon_{2} \geq 0$. The negative feedback interconnection of $\Sigma_{1}$ and $\Sigma_{2}$ is $\operatorname{IFP}(\kappa)$ with $\kappa=\min \left(\delta_{1}, \delta_{1}+\varepsilon_{2}\right)$.

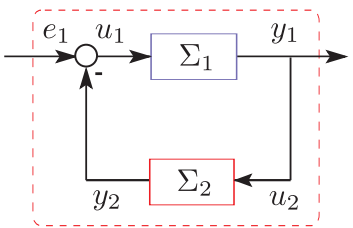

(a)

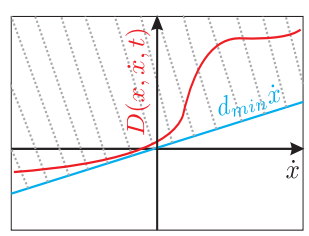

(b)
Fig. 2. (a) Negative feedback interconnection of systems $\Sigma_{1}$ and $\Sigma_{2}$. (b) Nonlinear time-varying damping with lower bound $d_{\min }$.

Proof : The compound storage function gives

$$
\begin{aligned}
& V(x(t))-V(x(0)) \leq \int_{0}^{t} e_{1}^{T} y_{1}+\left[\begin{array}{l}
e_{1} \\
y_{2}
\end{array}\right]^{T} W\left[\begin{array}{l}
e_{1} \\
y_{2}
\end{array}\right] \mathrm{d} \tau \\
& \leq \int_{0}^{t} e_{1}^{T} y_{1}+\lambda_{\max }(W)\left[\begin{array}{l}
e_{1} \\
y_{2}
\end{array}\right]^{T}\left[\begin{array}{l}
e_{1} \\
y_{2}
\end{array}\right] \mathrm{d} \tau,
\end{aligned}
$$

with

$$
W=\left[\begin{array}{cc}
-\delta_{1} I & 2 \delta_{1} I \\
0 & -\left(\delta_{1}+\varepsilon_{2}\right) I
\end{array}\right] .
$$

As $\lambda_{\max }(W)=-\kappa \leq 0$

$$
\begin{aligned}
V(x(t))-V(x(0)) & \leq \int_{0}^{t} e_{1}^{T} y_{1}-\kappa e_{1}^{T} e_{1}-\kappa y_{2}^{T} y_{2} \mathrm{~d} \tau \\
& \leq \int_{0}^{t} e_{1}^{T} y_{1}-\kappa e_{1}^{T} e_{1} \mathrm{~d} \tau,
\end{aligned}
$$

holds. Thus, the system is $\operatorname{IFP}(\kappa)$.

Among the variety of stability notions we consider finite gain $\mathscr{L}_{2}$-stability in this paper, which is another special case of quadratic dissipativity with $S=0, R=I, Q=-\gamma^{2} I$, $\gamma \in \mathscr{R}_{+}$.

Definition 2: [13] A dynamical system $\Sigma: \mathscr{U} \rightarrow \mathscr{Y}$, with $\mathscr{U} \subset \mathscr{L}_{2 e}^{m}$ representing the admissible input space and $\mathscr{Y}$ accordingly the output space is called finite gain $\mathscr{L}_{2}$-stable if there exists a constant $\gamma \in \Re$ such that for each $u \in \mathscr{U}$ and each $t \in[0, \infty)$

$$
\int_{0}^{t} y^{2} \mathrm{~d} \tau \leq \gamma \int_{0}^{t} u^{2} \mathrm{~d} \tau
$$

The smallest possible value $\gamma$ satisfying (2) is called the $\mathscr{L}_{2}$-gain of the system. An operator $\sigma: u(\cdot) \mapsto y(\cdot)$ with $u, y \in \mathfrak{R}^{p}$ the input and output respectively is called small gain operator if its $\mathscr{L}_{2}$-gain satisfies $\gamma_{\sigma} \leq 1$.

Finite gain $\mathscr{L}_{2}$-stability of a negative feedback interconnection can be concluded from the IF-OFP properties of its subsystems.

Proposition 1: [13] Consider two IF-OFP systems $\Sigma_{1}$ and $\Sigma_{2}$ with $\delta_{i}, \varepsilon_{i}, i \in 1,2$. The negative feedback interconnection of $\Sigma_{1}$ and $\Sigma_{2}$ is finite gain $\mathscr{L}_{2}$-stable if

$$
\varepsilon_{2}+\delta_{1}>0 \text { and } \varepsilon_{1}+\delta_{2}>0 .
$$

\section{MODELING OF TELEOPERATION SYSTEM}

In this paper we study a velocity-force teleoperation architecture. We assume that all the subsystems, human, environment, and manipulators, can be represented as dynamical systems in Cartesian space; for simplicity we consider only translation here. Hence, each subsystem is represented by the general structure

$$
M \ddot{x}(t)+D(x, \dot{x}, t)+K x(t)=f(t),
$$


where $x \in \Re^{n}$ is the position vector in the Cartesian space, $f \in \mathfrak{R}^{n}$ is the Cartesian force, $M \in \mathfrak{R}^{n \times n}$ and $K \in \mathfrak{R}^{n \times n}$ are the diagonal and positive-definite inertia and stiffness matrices, respectively. The components $D_{i}(x, \dot{x}, t)$ of the damping term $D(x, \dot{x}, t)=\operatorname{diag}\left\{D_{i}(x, \dot{x}, t)\right\}$ are assumed to be continuous, potentially time-varying and nonlinear functions for which $D(x, \dot{x}, t) \geq d_{\min } \dot{x}$, with $d_{\min } \geq 0$, holds componentwise and accounts for viscous damping, see Fig. 2(b) for a visualization in the 1-DoF case.

Recent works [3], [18], suggest that actual human arm endpoint characteristics are close to second-order; many nonactuated mechanical structures also have second-order structure. Note that for our approach we only have to assume that human and environment can be represented by a second order structure (3), however, without knowledge of the specific values of $M, D(\cdot)$, and $K$. Only knowledge on the lower bound of the damping represented by $d_{\min }^{h} \geq 0$ and $d_{\text {min }}^{e} \geq 0$ are required for human and environment.

For the controlled manipulators an impedance/admittance control scheme is considered; it can be used to render a desired dynamics or to achieve a certain compliant behavior to avoid contact instability, see e.g [12]. The impedance/admittance controlled manipulators, master and slave, are assumed articulated robots with position control in joint space and cartesian impedance. The joint controllers are assumed high gain; gravity and external forces are compensated, therefore the internal position loop dynamics can assumed to be negligible. The desired behavior is then achieved by implementing admittances in the form of simple mass-spring-damper system with linear time-invariant inertia, damping and stiffness matrices. The resulting master and slave dynamics is

$$
\begin{aligned}
f_{m}^{*}+f_{h}^{*}-f_{h} & =M_{m} \ddot{x}_{m}+D_{m} \dot{x}_{m}, \\
f_{e} & =M_{s} \ddot{x}_{s}+D_{s} \dot{x}_{s}+K_{s} x_{s}
\end{aligned}
$$

where $D_{i}$ the damping, $M_{i}$ inertia, $K_{i}$ stiffness, $x_{i}$ positions, and $i=\{m, s\}$ subscripts indicate master and slave, respectively. The human and environmental forces are represented by $f_{h}$ and $f_{e}$, respectively, whereas $f_{m}^{*}$ is the force-feedback term and $f_{h}^{*}$ the voluntarily applied force. The system structure is illustrated in Fig. 3. The blocks $\Xi$ and $\Xi^{-1}$ are explained in the next section.

With appropriate choice of the input/output pair the model (3) is input-feedforward-output-feedback passive (IF-OFP) system. Consider for example the force $f$ as input, the velocity $\dot{x}$ as output, and the following storage function

$$
V=\frac{1}{2} \dot{x}^{T} M \dot{x}+\frac{1}{2} x^{T} K x
$$

representing the kinetic and potential energy. Taking the derivative of it and integrating it follows

$$
\begin{aligned}
& V(x(t))-V(x(0))=\int_{0}^{t}(M \ddot{x}+K x)^{T} \dot{x} \mathrm{~d} \tau= \\
& =\int_{0}^{t}(f-D(x, \dot{x}, \tau))^{T} \dot{x} \mathrm{~d} \tau \leq \int_{0}^{t} f^{T} \dot{x}-d_{\min } \dot{x}^{T} \dot{x} \mathrm{~d} \tau
\end{aligned}
$$

The system is, hence, shown to be $\operatorname{OFP}\left(d_{\min }\right)$ with the input/output pair force/velocity and $d_{\min }=\varepsilon \geq 0$. Similarly,

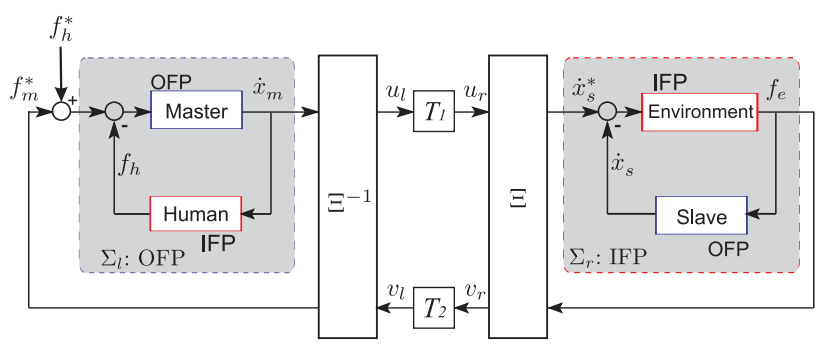

Fig. 3. Teleoperation system with time delay and generalized scattering transformation

the system can be shown to be $\operatorname{IFP}(\delta)$ with input $\dot{x}$, output $f$, and storage function (6)

$$
V(x(t))-V(x(0)) \leq \int_{0}^{t} \dot{x}^{T} f-d_{\min } \dot{x}^{T} \dot{x} \mathrm{~d} \tau,
$$

$d_{\min }=\delta \geq 0$.

Remark 1: For all subsystems, i.e. human, environment, and master and slave manipulator, knowledge of the lower sector bound of the damping force term $D(x, \dot{x}, t)$ of the system (3) is sufficient to guarantee IF-OFP properties. The inertia $M$ and stiffness $K$ components can be unknown as long as it can be shown that the subsystem has a second order structure as (3).

Remark 2: In case, where the bound of the damping force term $D(x, \dot{x}, t)$ of the system (3) is unknown, the analysis is reduced to the case of simply passive systems as performed in [1].

It is straightforward to see that that the systems (4) and (5) are subcases of the dynamics in (3) and the controlled manipulators are, hence, $\operatorname{OFP}\left(\varepsilon_{m}\right)$ and $\operatorname{OFP}\left(\varepsilon_{s}\right)$, respectively, with $\varepsilon_{m}=\lambda_{\min }\left(D_{m}\right)$ and $\varepsilon_{s}=\lambda_{\min }\left(D_{s}\right)$ being the smallest eigenvalues of the damping matrices. Accordingly with the choice of $\dot{x}_{m}$ as input and $f_{h}$ as output, the human arm is $\operatorname{IFP}\left(\delta_{h}\right)$ with $\delta_{h}=d_{\min }^{h}$. Similarly with input $\dot{x}_{s}^{*}-\dot{x}_{s}$ and output $f_{e}$, the environment is $\operatorname{IFP}\left(\delta_{e}\right)$ with $\delta_{e}=d_{\text {min }}^{e}$.

In Fig. 3, the feedback interconnection structure of the considered velocity-force architecture is illustrated. A small gain network operator represents the communication unreliability in the forward and feedback path, it can be constant, arbitrarily large time delay as visualized in Fig. 3.

Proposition 2: The negative feedback interconnection of the lefthand subsystem $\Sigma_{l}$ in Fig. 3, with $f_{m}^{*}+f_{h}^{*}$ as input and $\dot{x}_{m}$ as output is $\operatorname{OFP}\left(\varepsilon_{l}\right)$ with $\varepsilon_{l}=\varepsilon_{m}+\delta_{h} \geq 0$. Similarly, the negative feedback interconnection of the righthand subsystem $\Sigma_{r}$, with $\dot{x}_{s}^{*}$ as input and $f_{e}$ as output is $\operatorname{IFP}\left(\delta_{r}\right)$ with $\delta_{r}=\min \left(\delta_{e}, \delta_{e}+\varepsilon_{s}\right)$.

Proof: Straightforward from Lemma 1 and Lemma 2.

In general, from now on we consider the networked interconnection of an $\operatorname{OFP}\left(\varepsilon_{l}\right)$ and an $\operatorname{IFP}\left(\delta_{r}\right)$ system with $\varepsilon_{l}, \delta_{r}>0$ with

$$
P_{l}=\left[\begin{array}{cc}
0 & \frac{1}{2} I \\
\frac{1}{2} I & -\varepsilon_{l} I
\end{array}\right], P_{r}=\left[\begin{array}{cc}
-\delta_{r} I & \frac{1}{2} I \\
\frac{1}{2} I & 0
\end{array}\right]
$$

their corresponding dissipativity matrices. 
Remark 3: Straightforwardly, this analysis can be carried out for other teleoperation architectures, e.g. the forcevelocity architecture.

\section{GENERALIZED SCATTERING TRANSFORMATION}

The generalized scattering transformation is a linear input/output transformation to guarantee stability in presence of any small gain operator in the communication loop and it is represented by the matrix $\Xi$ in Fig. 3. Instead of the lefthand output variable $\dot{x}_{m}$ the variable $u_{l}$ is transmitted.

$$
\left[\begin{array}{l}
u_{l} \\
v_{l}
\end{array}\right]=\Xi\left[\begin{array}{c}
\dot{x}_{m} \\
f_{m}^{*}
\end{array}\right] \text {. }
$$

Analogously, $v_{r}$ is transmitted instead of $f_{e}$ where

$$
\left[\begin{array}{c}
u_{r} \\
v_{r}
\end{array}\right]=\Xi\left[\begin{array}{c}
\dot{x}_{s}^{*} \\
f_{e}
\end{array}\right] .
$$

The $u_{r}$ and $v_{l}$ are the output of the network operator for the forward and backward channel, respectively. The transformation is parametrized using a rotation matrix $R$ and a scaling matrix $B$

$$
\Xi=R \cdot B=\left[\begin{array}{cc}
\cos \theta I & \sin \theta I \\
-\sin \theta I & \cos \theta I
\end{array}\right]\left[\begin{array}{cc}
b_{11} I & 0 \\
0 & b_{22} I
\end{array}\right]
$$

where $I$ represents the $n \times n$ unity matrix, $\operatorname{det} B \neq 0$ and $\theta \in\left[-\frac{\pi}{2}, \frac{\pi}{2}\right]$. The choice of the transformation angle $\theta$ is based on the IFP- and OFP-properties of each side, $b_{11}, b_{22}>0$ represent free tuning parameters.

Proposition 3: [10] Assume a system consisting of the networked negative feedback interconnection of an $\operatorname{OFP}\left(\varepsilon_{l}\right)$ and an $\operatorname{IFP}\left(\delta_{r}\right)$ system with $\varepsilon_{l}, \delta_{r}>0$, the bidirectional communication channel and the input-output transformation (7). Finite gain $\mathscr{L}_{2}$-stability is ensured for any small gain operator in the network if and only if for each $B$ the rotation matrix parameter $\theta \in\left[\theta_{l}, \theta_{r}\right]$. Here $\theta_{l}$ and $\theta_{r}$ are one of the two solutions of

$$
\cot 2 \theta_{i}=\varepsilon_{B_{i}}-\delta_{B_{i}}, i \in\{l, r\}
$$

which simultaneously satisfy

$$
a\left(\theta_{i}\right)=\sin \theta_{i} \cos \theta_{i}-\delta_{B_{i}} \cos ^{2} \theta_{i}-\varepsilon_{B_{i}} \sin ^{2} \theta_{i} \geq 0,
$$

$\varepsilon_{B_{i}}$ and $\delta_{B_{i}}$ are given by the matrix $P_{B_{i}}$

$$
\begin{aligned}
P_{B_{i}} & =\left[\begin{array}{cc}
-\delta_{B_{i}} I & \frac{1}{2} I \\
\frac{1}{2} I & -\varepsilon_{B_{i}} I
\end{array}\right]=B^{-T} P_{i} B^{-1}= \\
& =\left[\begin{array}{cc}
-b_{22}^{2} \delta_{i} I & -\frac{1}{2} b_{11} b_{22} I \\
-\frac{1}{2} b_{11} b_{22} I & -b_{11}^{2} \varepsilon_{i} I
\end{array}\right], i \in\{l, r\} .
\end{aligned}
$$

Hence, instead of choosing $\theta=45^{\circ}$ and $b_{11}=\sqrt{b}, b_{22}=\frac{1}{\sqrt{b}}$, as for standard scattering transformation [1], here $\theta$ can be chosen out of an interval.

Remark 4: Finite gain $\mathscr{L}_{2}$-stability of the whole system is guaranteed for any small gain operator in the communication loop. It is straightforward to see that this applies not only to arbitrarily large constant time delay, which has a $\mathscr{L}_{2}$-gain $\gamma_{D}=1$, but also for time-varying delay [4] and properly handled packet loss [2], [7], as the passivity-preserving algorithms presented have a $\mathscr{L}_{2}$-gain $\gamma_{P} \leq 1$.

\section{TRANSPARENCY ANALYSIS}

Transparency is the ability of the teleoperation system to present the undistorted dynamics of the environment to the human. As a result the human feels like "being" on the remote side and directly interacting with the environment. Modeling the real environment as a mechanical linear timeinvariant impedance $Z_{e}(s)$ and the impedance displayed to the human $Z_{h}(s)$, transparency is achieved if [14]

$$
Z_{h}(s)=Z_{e}(s),
$$

where $s$ is a complex variable, representing the Laplace domain; it will be omitted when not needed.

In practice, perfectly transparent teleoperation is difficult to achieve. The interesting question is the degree of transparency that is possible on a teleoperation system. Ignoring the controller dynamics and robot compliance we investigate in this section the case of constant time delay. The displayed impedance can be computed based on the environment impedance and the generalized scattering transformation according to

$$
Z_{h}=\frac{\xi_{21}-\xi_{11} \operatorname{Re}^{-} s T}{-\xi_{22}+\xi_{12} \operatorname{Re}^{-} s T}, R=\frac{\xi_{21}+\xi_{22} Z_{e}}{\xi_{11}+\xi_{12} Z_{e}},
$$

where $T=T_{1}+T_{2}$ the round trip time delay and

$$
\Xi=\left[\begin{array}{ll}
\xi_{11} & \xi_{12} \\
\xi_{21} & \xi_{22}
\end{array}\right] \text {. }
$$

In order to analyze and compare different impedances a Padé approximation will be used for the time-delay, which is, however, only valid for low frequencies, i.e. $e^{-s T} \approx\left(1-\frac{T}{2} s\right) \cdot\left(1+\frac{T}{2} s\right)^{-1}$ for $\omega<\frac{1}{3 T}$. The teleoperator dynamics are ignored for simplicity. The transformation angle is limited to $\theta \in\left[0, \frac{\pi}{2}\right]$.

The displayed impedance in case of an environment with a spring and a damper $Z_{e}=\frac{k_{e}}{s}+b_{e}$ is approximated for low frequencies by (11) as

$$
\left.Z_{h}^{L F}\right|_{Z_{e}=\frac{k_{e}}{s}+b_{e}}=\frac{\gamma_{\frac{k_{e}}{s}}+b_{h}+m_{h} s}{1+\gamma\left(\frac{T}{2}-\frac{b_{22}}{b_{11}} T b_{e} \sin \theta \cos \theta\right) s}
$$

with $\gamma=\frac{1}{1+T k_{e} \sin \theta \cos \theta}, \quad b_{h}=\gamma\left(b_{e}+\frac{T}{2} k_{e}\left(\sin ^{2} \theta-\cos ^{2} \theta\right)\right)$, $m_{h}=\gamma\left(\frac{b_{11}}{b_{22}} T \sin \theta \cos \theta+\frac{T}{2} b_{e}\left(\sin ^{2} \theta-\cos ^{2} \theta\right)\right) \quad$ and $\frac{b_{11}}{b_{22}}>2 b_{e} \sin \theta \cos \theta$; the latter resulting from the requirement on stable transfer functions in the approximation. The denominator of (12) can be shown to be a low pass filter which in the worst case, i.e. $\theta=0^{\circ}$ or $\theta=90^{\circ}$, has a cut-off frequency $\frac{2}{T}>\frac{1}{3 T}$, therefore, it can be ignored. The resulting displayed impedance is then

$$
\left.Z_{h}^{L F}\right|_{Z_{e}=\frac{k_{e}}{s}+b_{e}}=\gamma \frac{k_{e}}{s}+b_{h}+m_{h} s
$$

Similarly, it can be shown that for free space motion an inertia linearly increasing with the time delay is approximately displayed

$$
m_{h} \approx \frac{b_{11}}{b_{22}} T \sin \theta \cos \theta .
$$

The computation is straightforward from (11) with $Z_{e}=0$. 


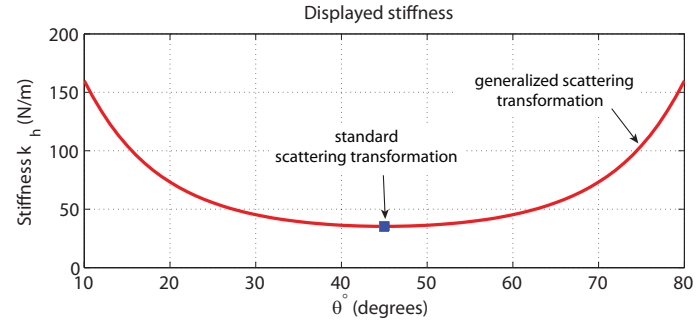

Fig. 4. Displayed stiffness $k_{h}$ depending on rotation angle $\theta$, environment stiffness is $300 \mathrm{~N} / \mathrm{m}$, damping is $30 \mathrm{Ns} / \mathrm{m}$, and $B=I$.

By (13) and (14) it can be derived that the scaling matrix alters the performance of the system in a similar way the characteristic impedance configures the performance of the scattering transformation architecture, cf. [8]. Considering the low frequency approximation, a small factor $\frac{b_{11}}{b_{22}}$ will avoid large inertia in free space movement whereas a large one is required to display high stiffness. Moreover, it can be seen in (8), (9) and (10) that the choice of the scaling components influences the region $\left[\theta_{l}, \theta_{r}\right]$ that finite gain $\mathscr{L}_{2}$ stability can be guaranteed. An increasing factor $\frac{b_{11}}{b_{22}}$ will allow for lower $\theta_{l}$ whereas if $\frac{b_{11}}{b_{22}}$ is decreased, the interval $\left[\theta_{l}, \theta_{r}\right]$ is increasing from the righthandside, i.e. higher $\theta_{r}$.

In order to exemplarily compare the standard scattering transformation with the generalized scattering transformation we choose the scaling components of the generalized scattering transformation such that the displayed inertia in free space (14) is the same as for the standard scattering transformation, namely $\frac{b_{11}}{b_{22}}=\frac{b}{2 \sin \theta \cos \theta}$. The approximated displayed stiffness $k_{h}$ is then compared for the two methods, see Fig. 4, and it is seen that the stiffness of the generalized scattering transformation outperforms the one displayed by the conventional scattering transformation approach, i.e. for $\theta=45^{\circ}$ and the choice $b=1$. The bigger the deviation from the $45^{\circ}$ the larger the improvement in terms of displayed stiffness, however, the damping properties are slightly distorted (13).

The proposed approach is demonstrated in simulation for a teleoperation velocity-force control scheme with a linear time-invariant spring-damper environment $Z_{e}=\frac{300}{s}+30$, hence $\delta_{r}=\delta_{e}=30$, and negligible slave dynamics. The lefthandside system is considered $\operatorname{OFP}\left(\varepsilon_{l}\right)$ with $\varepsilon_{l}=10$ resulting from either the human or master dynamics' minimum damping. The two systems result in dissipativity matrices $P_{r}=\left[\begin{array}{cc}-30 & 1 / 2 \\ 1 / 2 & 0\end{array}\right]$ and $P_{l}=\left[\begin{array}{cc}0 & 1 / 2 \\ 1 / 2 & -10\end{array}\right]$. For comparison reasons a characteristic impedance $b=1$ is chosen for the scattering transformation and the generalized scattering transformation is tuned with scaling components, $b_{11}=\sqrt{b}=1$ and $b_{22}=\frac{2 b_{11} \sin \theta \cos \theta}{b}$ such that in free space both methods display same inertia. The time delay is $T_{1}=T_{2}=50 \mathrm{~ms}$ and the resulting system is according to Prop. 3 delay-independently stable for all $\theta \in\left[3^{\circ}, 87^{\circ}\right]$. In Fig. 5 we observe, that the generalized scattering transformation outperforms the standard scattering transformation. Particularly for the case where $\theta=\left\{30^{\circ}, 60^{\circ}\right\}$ a stiffness of
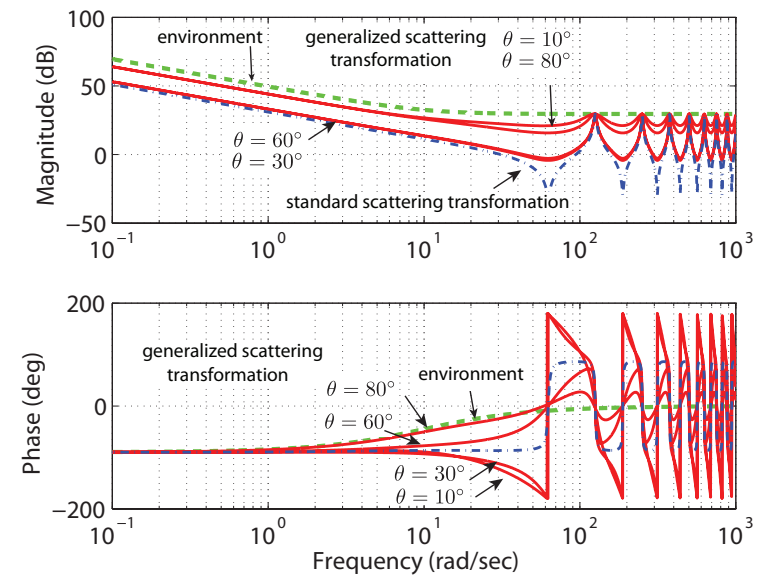

Fig. 5. Displayed impedance comparison in contact with the environment, $Z_{e}=\frac{300}{s}+30$.

45.7 N/m is displayed, whereas if $\theta=\left\{10^{\circ}, 80^{\circ}\right\}$ a stiffness $k_{h}=\{164.4,166.3\} \mathrm{N} / \mathrm{ms}$ displayed. This by far closer to the real environment stiffness of $k_{e}=300 \mathrm{~N} / \mathrm{m}$ than the scattering transformation which displays only $35.5 \mathrm{~N} / \mathrm{m}$, see Fig. 5. The structural difference between the two symmetric choices $\theta=\frac{\pi}{4} \pm \Delta, \Delta \in\left[0, \frac{\pi}{4}\right]$ is currently under investigation.

\section{EXPERIMENTAL EVALUATION}

The goal of this section is to validate the proposed approach in an experiment. A human interacts with a virtual environment using a teleoperation system over a communication channel, and the displayed impedance is computed to evaluate the approach. The experimental testbed consists of two tubular linear motors, Thrusttube 2510 from Copley Controls Corp., shown in Fig. 6, two force sensors from Burster Corp. and a PC. Each linear motor is able to display peak forces of up to $780 \mathrm{~N}$ and $104.3 \mathrm{~N}$ of continuous stall forces and is connected to a digital servo drive Xenus XTL, again from Copley Controls Corp. The digital servo is operated in current control, thus, we can consider the signal input to be approximately proportional to the applied motor force. The position is measured by an optical incremental encoder with a precision of $1 \mu \mathrm{m}$. The whole haptic interface is controlled from a PC running a real time Linux operating system. The digital servo is connected to the PC through a Sensoray I/O card. All the control functions are implemented by Simulink blocksets including friction compensation and velocity computation. The applied sampling rate of the haptic signals and the local control loops is $1000 \mathrm{~Hz}$.

A virtual linear time-invariant spring-damper environment $Z_{e}=\frac{300}{s}+30$, i.e. $d_{\min }^{e}=30$. A position-based admittance control scheme was used, with master impedance $Z_{m}=3 s+10$. Therefore, a lower master damping $\varepsilon_{m}=d_{\min }^{m}=10$ could be guaranteed to experimentally test the algorithm. The scaling parameters were chosen such that the free-space motion displayed dynamics are similar in both methods, $b=70, b_{11}=\sqrt{70}, b_{22}=\frac{2 b_{11} \sin \theta \cos \theta}{b}$ and resulted in $\theta_{l}=2^{\circ}$ and $\theta_{r}=55^{\circ}$. The proposed scheme was tested initially with an angle $\theta=52^{\circ}$. The 


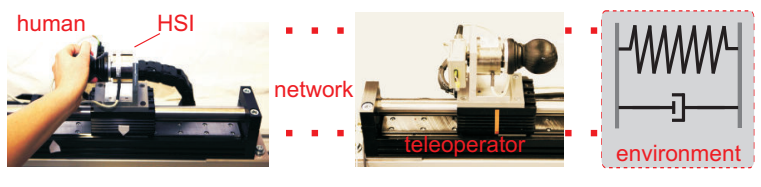

Fig. 6. Experimental setup
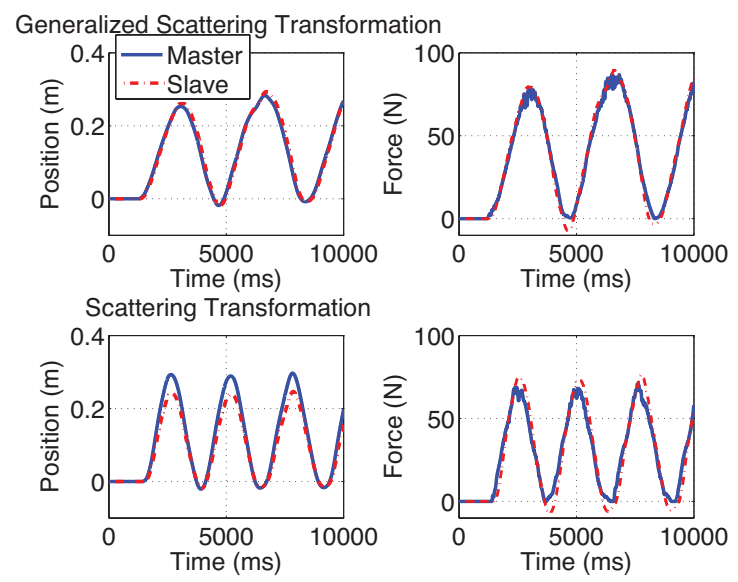

Fig. 7. Position and force tracking

time delay was $50 \mathrm{~ms}$ in both channels. The system was stable throughout the experiment. The displayed impedance is identified, using a least-square identification. For the generalized scattering transformation a displayed impedance of $k_{h}=245 \mathrm{~N} / \mathrm{m}, b_{h}=31 \mathrm{Ns} / \mathrm{m}$ is identified, which is slightly improved compared to the displayed stiffness of the scattering transformation, $k_{h}=228 \mathrm{~N} / \mathrm{m}, b_{h}=26 \mathrm{Ns} / \mathrm{m}$. Starting oscillations did not allow for further increase of $\theta$. For $\theta=11^{\circ}$ the position and force signals are illustrated in Fig. 7. The displayed impedance was again identified, for the generalized scattering transformation $k_{h}=302 \mathrm{~N} / \mathrm{m}, \quad b_{h}=1.1 \mathrm{Ns} / \mathrm{m} \quad$ obviously outperforming compared to the displayed stiffness achieved with the scattering transformation. Experiments showed that, the closer to the marginal condition, i.e. $\theta_{l}=2^{\circ}$, the system was tuned, the more the system compensated its extra damping for a higher displayed stiffness, see (13).

\section{CONCLUSIONS}

In this paper, approximate knowledge on the damping of the human, the environment or/and the controlled manipulators of a teleoperation scheme is used in order to improve the performance. The subsystems can then be shown to be modeled as IF-OFP systems, a subclass of QSR-dissipative systems. This allows the application of the generalized scattering transformation guaranteeing finite gain $\mathscr{L}_{2}$-stability for all small gain operators in the communication loop. Transparency analysis for the constant time delay case shows the superior performance of the proposed approach. The proposed approach is validated in simulation studies and experiments on a 1-DoF teleoperation system.

\section{ACKNOWLEDGMENTS}

This work is supported by the German Research Foundation (DFG) within the Collaborative Research Center SFB453 on "High-Fidelity Telepresence and Teleaction" and the Priority Program SPP 1305 "Control Theory of Digitally Networked Dynamical Systems". The constructive comments by the anonymous reviewers are highly appreciated.

\section{REFERENCES}

[1] R. J. Anderson and M. W. Spong. Bilateral control of teleoperators with time delay. IEEE Transactions on Automatic Control, 34(5):494 501, May 1989

[2] P. Berestesky, N. Chopra, and M. W. Spong. Discrete time passivity in bilateral teleoperation over the internet. In Proceedings of the 2004 IEEE International Conference on Robotics and Automation, ICRA '04, volume 5, pages 4557 - 4564, April 2004.

[3] S. Buerger and N. Hogan. Relaxing passivity for human-robot interaction. In Proceedings of the IEEE/RSJ International Conference on Intelligent Robots and Systems, pages 4570-4575, October 2006.

[4] N. Chopra, M. Spong, S. Hirche, and M. Buss. Bilateral teleoperation over the internet: the time varying delay problem. In American Control Conference, 2003. Proceedings of the 2003, volume 1, pages 155-160, 4-6 June 2003

[5] N. Diolaiti, C. Melchiorri, and S. Stramigioli. Contact impedance estimation for robotic systems. Robotics, IEEE Transactions on, 21(5):925 - 935, oct. 2005.

[6] R. Gillespie and M. Cutkosky. Stable user-specific haptic rendering of the virtual wall. In Proceedings of the International Mechanical Engineering Comgress and Exhibition, volume 61, pages 85-92, November 1995.

[7] S. Hirche and M. Buss. Packet loss effects in passive telepresence systems. In Decision and Control, 2004. CDC. 43rd IEEE Conference on, volume 4, pages 4010-4015 Vol.4, Dec. 2004.

[8] S. Hirche and M. Buss. Human perception oriented control aspects of networked telepresence and teleaction systems. In Proceedings of the International Conference on Instrumentation, Control and Information Technology, pages 3430-3435, Okayama, Japan, 2005.

[9] S. Hirche, M. Ferre, J. Barrio, C. Melchiorri, and M. Buss. Bilateral Control Architectures for Telerobotics, chapter Control, pages 163176. STAR. Springer-Verlag, Berlin, 2007.

[10] S. Hirche, T. Matiakis, and M. Buss. A distributed controller approach for delay-independent stability of networked control systems. Automatica, 45(8):1828 - 1836, 2009.

[11] N. Hogan. Controlling impedance at the man/machine interface. In Robotics and Automation, 1989. Proceedings., 1989 IEEE International Conference on, volume 3, pages 1626-1631, Scottsdale, AZ, 14-19 May 1989.

[12] N. Hogan. Impedance control: An approach to manipulation, part i - theory, part ii - implementation, part iii - applications. Journal of Dynamical Systems, Measurements, and Control, 107:1-24, 1985.

[13] H. K. Khalil. Nonlinear Systems. Prentice Hall, 1996.

[14] D. Lawrence. Stability and transparency in bilateral teleoperation. Decision and Control, 1992., Proceedings of the 31st IEEE Conference on, pages 2649-2655 vol.3, 1992.

[15] T. Matiakis, S. Hirche, and M. Buss. Independent-of-delay stability of nonlinear networked control systems by scattering transformation. American Control Conference, 2006, pages 2801-2806, June 2006.

[16] T. Matiakis, S. Hirche, and M. Buss. Networked control systems with time-varying delay - stability through input-output transformation. Automatisierungstechnik, 56(1):29-37, 2008

[17] G. Niemeyer and J.-J. E. Slotine. Stable adaptive teleoperation. IEEE Journal of Oceanic Engineering, 16(1):152-162, January 1991.

[18] T. Tsuji, P. Morasso, K. Goto, and K. Ito. Human hand impedance characteristics during maintained posture. Biological Cybernetics, 72(6):475-485, May 1995.

[19] J. C. Willems. Dissipative dynamical systems part i: General theory. Archive for Rational Mechanics and Analysis, 45(5):321 - 351, January 1972.

[20] Y. Yokokohji, T. Tsujioka, and T. Yoshikawa. Bilateral control with time-varying delay including communication blackout. In Haptic Interfaces for Virtual Environment and Teleoperator Systems, International Symposium on, volume 0, page 285, Los Alamitos, CA, USA, 2002. IEEE Computer Society. 